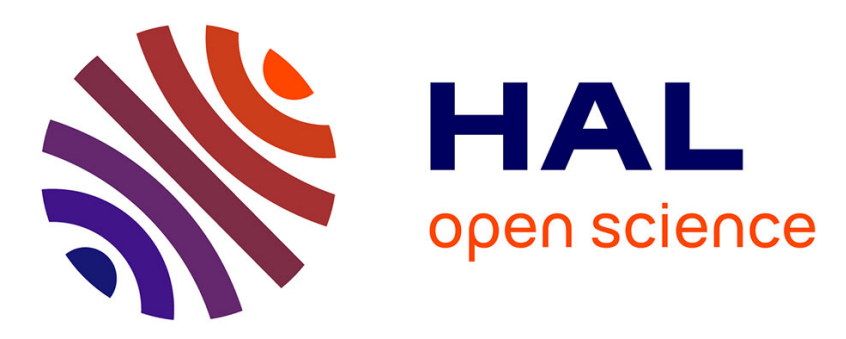

\title{
New Damped-Jerk trajectory for vibration reduction
} Richard Béarée

\section{To cite this version:}

Richard Béarée. New Damped-Jerk trajectory for vibration reduction. Control Engineering Practice, 2014, 28, pp.112-120. 10.1016/j.conengprac.2014.03.010 . hal-01064647

\section{HAL Id: hal-01064647 https://hal.science/hal-01064647}

Submitted on 16 Sep 2014

HAL is a multi-disciplinary open access archive for the deposit and dissemination of scientific research documents, whether they are published or not. The documents may come from teaching and research institutions in France or abroad, or from public or private research centers.
L'archive ouverte pluridisciplinaire $\mathbf{H A L}$, est destinée au dépôt et à la diffusion de documents scientifiques de niveau recherche, publiés ou non, émanant des établissements d'enseignement et de recherche français ou étrangers, des laboratoires publics ou privés. 


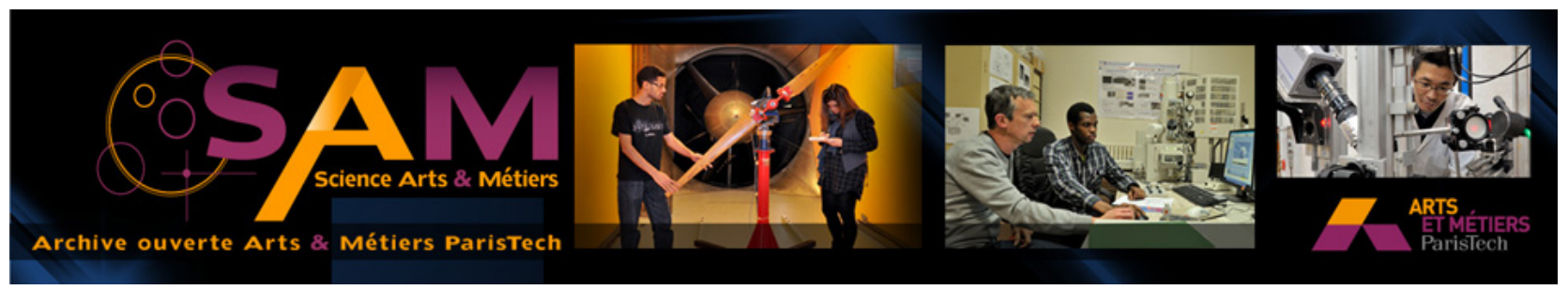

Science Arts \& Métiers (SAM)

is an open access repository that collects the work of Arts et Métiers ParisTech researchers and makes it freely available over the web where possible.

This is an author-deposited version published in: http://sam.ensam.eu Handle ID: .http://hdl.handle.net/10985/8532

\section{To cite this version :}

Richard BEAREE - New Damped-Jerk trajectory for vibration reduction - Control Engineering Practice - Vol. 28, p.112-120 - 2014 


\section{Richard Béarée}

Arts et Métiers ParisTech, LSIS, 8 Bd Louis XIV, 59046 Lille, France

\section{Keywords:}

Vibration

Damped frequency

Command signal

Shaping filters

Trajectories

\begin{abstract}
A B S T R A C T
This paper derives a jerk-shaped profile to address the vibration reduction of underdamped flexible dynamics of motion system. The jerk-limited profile is a widespread smooth command pattern used by modern motion systems. The ability of the jerk-limited profile to cancel the residual vibration of an undamped flexible mode is clearly explained using an equivalent continuous filter representation and the input shaping formalism. This motivates the design of a new jerk-shaped profile, named DampedJerk profile, to extend the previous result to the more common case of underdamped systems. Both simulations and experimental results demonstrate the effectiveness of the proposed DampedJerk profile to reduce damped vibration.
\end{abstract}

\section{Introduction}

The jerk-limited profile is a widespread trajectory pattern used by modern motion systems, such as mobile robots, machine-tools or industrial robots. Fig. 1 depicted such a profile, which is a time optimal solution to the problem of jerk limited rigid body control. The jerk limitation (the slope or time derivative of acceleration) is classically considered as a smoothness parameter, which is empirically used for the limitation of deformations and vibrations induced by the reference trajectory.

It is recognized that if the main vibration is not caused by external disturbances and if the flexible dynamics of the system is known, reference trajectory can be generated to cancel or reduce the vibration. One solution consists in defining a suitable parameterized trajectory, which assures that no oscillation occurs during and at the end of the motion. Such a method is mainly based on a system inversion principle. In Piazzi and Visioli (2000), this method appears to be very effective in reducing the residual vibration, and the smoothness of the control input makes it inherently robust to modeling errors. In Kim and Agrawal (2006), both the simulation and experimental results are presented to show the effectiveness of various jerk limited control profiles to reduce the vibration of a flexible structure. The authors state that the jerk limited rigid-body control profile such as versine or polynomial profile is effective to reduce the excitation of the flexible modes, however, better performance can be obtained from the handling of the low frequency modes as in an input shaped command. Input shaping is another known command generation methodology to suppress vibration, as detailed in Smith (1957), Singer and Seering (1990), Singhose and Pao (2009) and La-orpacharapan and Pao (2005). Input shaping relies on the convolution of a sequence of impulses with the reference signal. This convoluted signal is then used as a new reference for the system. Hence, vibrations induced by the first impulse is canceled or reduced at the end of the sequence. Input shaping has been implemented with success on numerous systems, and more specifically on manipulators and gantry cranes (Diaz, Pereira, Feliu, \& Cela, 2010; Park, Chang, Park, \& Lee, 2006; Peng, Singhose, \& Frakes, 2012).

In Olabi, Béarée, Gibaru, and Damak (2010) and Béarée and Olabi (2013), we demonstrate that the jerk-limited profile can be used to cancel the residual vibration of an undamped system. The jerk time (i.e. the acceleration slope time) can be explicitly linked to the vibration caused by a dominating flexible mode. Hence, the jerk-limited profile can be seen as a special combination of the smoothness property of polynomial profiles and the vibration reduction property of basic input shaping. However, these elegant properties vanish when the damping coefficient of the considered flexible mode is not negligible. In this paper, we propose a simple modification of the jerk-limited profile in view of generalizing the previous property to the case of underdamped flexible mode. The resulting profile keeps the generic aspect, the easiness of implementation and the simplicity of tuning of the original jerk-limited profile, which are the main motivations of this study.

The outline of this paper is as follows: In Section 2 we present clear explanation regarding the ability of the jerk-limited profile to cancel the residual vibration for an undamped flexible mode and show how this profile can be characterized using input shaping 
and continuous time filter formalism. We then proposed, in Section 3, a modified Damped-Jerk profile. In this section, the new profile is analyzed and compared to the original jerk-limited profile. In Section 4, experimental measurements conducted on an industrial robot confirm that the Damped-Jerk profile is an effective and elegant solution to the problem of underdamped vibration reduction.

\section{Jerk-limited trajectory and vibration reduction}

\subsection{Input shaping formalism adapted to the jerk-limited profile}

As depicted in Fig. 1, a jerk-limited profile implies a trapezoidal or a triangular acceleration profile. Such a trajectory can be easily and efficiently synthesized by applying a moving averaging FIR filter to an acceleration limited profile (sometimes called bang-offbang profile), as described in Biagiotti and Melchiorri (2012) and Singh (2004). The filter time, equivalent to the jerk time noted $T_{J}$, is fixed by the relation between the maximum acceleration value and the maximum jerk value given by

$T_{J}=A_{\max } / J_{\max }$.

In the context of the trajectory following with kinematic constraints on acceleration and velocity, the filter introduces a time delay, which could have a detrimental effect on the accuracy of the new reference trajectory. In Béarée and Olabi (2013) a simple adaptation of the initial acceleration-limited trajectory is proposed to take account of the filter time. One notes that this methodology reduces significantly the algorithm complexity of a classical jerklimited trajectory generator. Hence, a jerk-limited profile can be seen as the convolution of a simple averaging filter, noted $F_{J L}$, with an adapted acceleration-limited profile. In the same manner, the averaging filter can be perceived as the convolution of an integrator with a succession of two impulses given in continuous time domain by the transfer function

$F_{J L}(s)=\frac{1}{s}\left(A_{1}+A_{2} e^{-s T_{J}}\right)$,
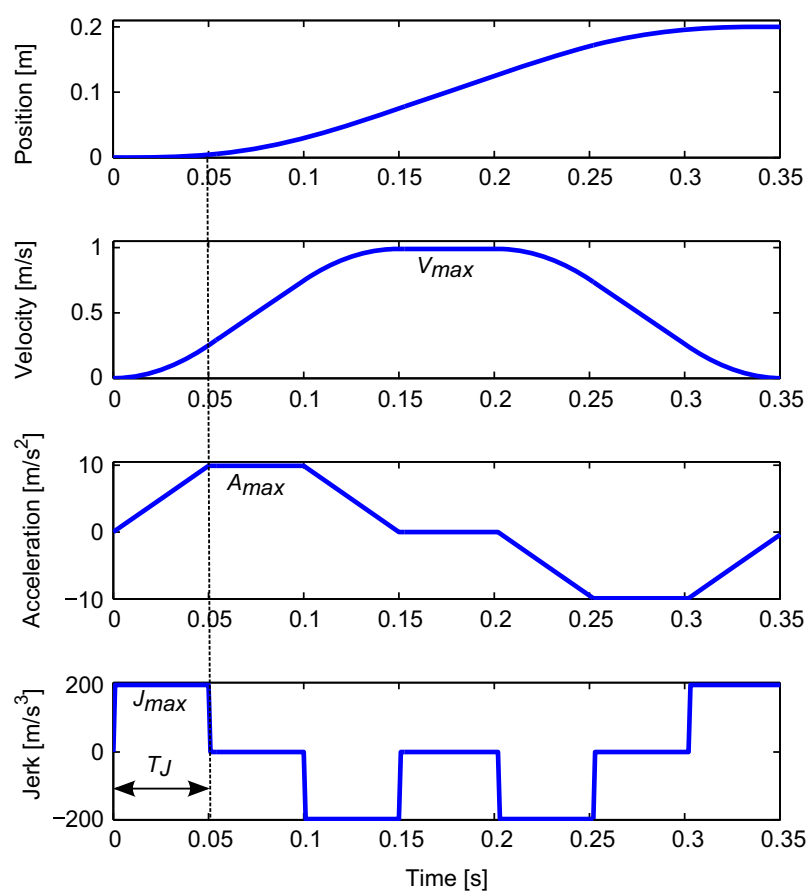

Fig. 1. Example of a jerk-limited rest-to-rest profile.

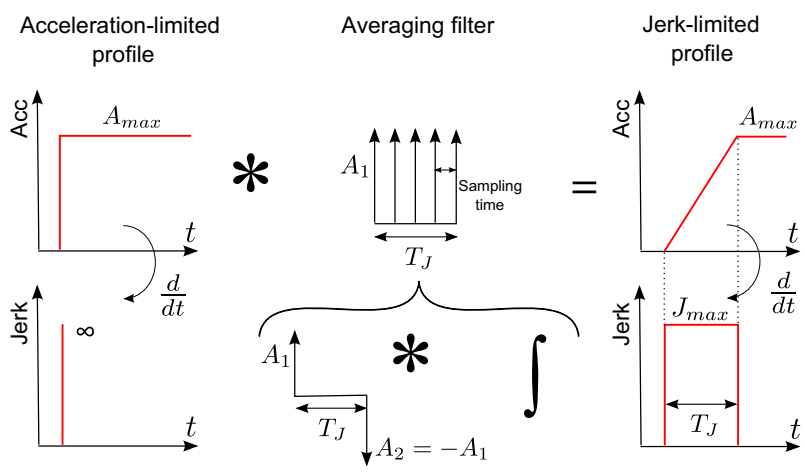

Fig. 2. Smooth input shaper associated to the jerk-limited profile.

with $A_{1}=-A_{2}=1 / T_{J}$. One notes that for discrete time implementation, $T_{J}$ will be an integral multiple of the sampling interval.

Finally, as depicted in Fig. 2, the jerk-limited profile can be interpreted as a special case of a smooth negative input shaper. The smoothness is given by the low-pass filtering effect of the integrator and the negative input shaper part is the key explaining the vibration reduction ability of the jerk-limited profile. Considering a system submitted to a dominant undamped flexible mode at a frequency of $\omega_{1}$, the cancellation of the residual vibration by the jerk-limited profile is equivalent to the zeros placement at the undamped flexible poles locations, i.e. at $s= \pm j \omega_{1}$. Applying this principle to the previous jerk shaper given by (2), the resulting equation to be solved becomes

$\left.F_{J L}(s)\right|_{s= \pm j \omega_{1}}=0$.

This expression can be rewritten as a system of trigonometric equations

$A_{1}+A_{2} \cos \left(\omega_{1} T_{J}\right)=0$

$\sin \left(\omega_{1} T_{J}\right)=0$

A trivial solution to (4) is then given by

$T_{J}=k \frac{2 \pi}{\omega_{1}}$

$A_{1}+A_{2}=0$

with $k$ being a positive integer. To keep the constraint imposed on the initial acceleration profile, the integral of the shaper has to be equal to 1 , which conduct to the following set of solutions:

$T_{J}=k \frac{2 \pi}{\omega_{1}}$

$A_{1}=-A_{2}=\frac{1}{T_{J}}$

Based on the input shaping formalism, the previous result concludes the explanation about the capacity of jerk-limited to cancel the residual vibration for an undamped flexible mode. The jerk time can be taken equal to a multiple integer of the natural period of vibration. For time minimization, the jerk time is classically chosen equal to the natural period of the dominating flexible mode. Using this tuning methodology the maximum jerk value will be imposed by the jerk time and the maximum acceleration value according to the relation (1). The vibration reduction ability of such a strategy is sensitive to modeling errors or uncertainty on the frequency of the flexible mode. To clearly measure this effect, the amplitude of residual vibration can be plotted as a function of modeling errors. Such a plot is classically called a sensitivity curve. 


\subsection{Jerk-limited profile sensitivity curve}

Here we need an expression for the residual vibration amplitude of the jerk-limited profile as a function of the filter time or the frequency. The residual vibration can be calculated using the expression for residual vibration of a second-order harmonic oscillator of frequency $\omega_{1}$ and damping ratio $\zeta$, which is detailed in Singhose (2009). The response from a sequence of impulses, noted $y_{\text {imp }}(t)$, is the superposition of individual impulse responses

$y_{\text {imp }}(t)=\sum_{i=1}^{n} \frac{A_{i} \omega_{1}}{\sqrt{1-\zeta^{2}}} e^{-\zeta \omega_{1}\left(t-T_{i}\right)} \sin \left(\omega_{1 d}\left(t-T_{i}\right)\right)$,

with $A_{i}$ and $T_{i}$ being the amplitude and commutation time of each impulse, respectively, and $\omega_{1 d}=\omega_{1} \sqrt{1-\zeta^{2}}$ the damped frequency. Using trigonometric identities and noting $T$ as the time length of the sequence of impulse, the residual vibration amplitude at time $T$ divided by the vibration from a single unity-magnitude impulse to get the percentage of vibration can be expressed as

$V\left(\omega_{1}\right)=e^{-\zeta \omega_{1} T} \sqrt{C^{2}+S^{2}}$,

with

$C=\sum_{i=1}^{n} A_{i} e^{\zeta \omega_{1} T_{i}} \cos \left(\omega_{1 d} T_{i}\right)$,

$S=\sum_{i=1}^{n} A_{i} e^{\zeta \omega_{1} T_{i}} \sin \left(\omega_{1 d} T_{i}\right)$

The residual vibration for one stage of constant jerk (see Fig. 2) is deduced from the decomposition into a negative shaper given by (6) and an integrator, which corresponds to a division by $\omega_{1}$ for the amplitude of vibration. Thus, the residual vibration $V_{J L}$ for a jerk-limited profile can be expressed as

$V_{J L}\left(\omega_{1}\right)=\frac{e^{-\zeta \omega_{1} T_{J}}}{\omega_{1} T_{J}} \sqrt{1+e^{2 \zeta \omega_{1} T_{J}}-2 e^{\zeta \omega_{1} T_{J}} \cos \left(\omega_{1 d} T_{J}\right)}$.

Assuming an undamped mode $(\zeta=0)$ and noting $\omega_{0}=2 \pi / T_{J}$, (11) is rewritten

$V_{J L}\left(\omega_{1}\right)=\frac{\sin \left(\pi \omega_{1} / \omega_{0}\right)}{\left(\pi \omega_{1} / \omega_{0}\right)}$.

According to (12), residual vibration for an undamped mode is a sine cardinal function of the frequency. Residual vibration for an undamped flexible mode will be suppressed for a jerk time equal to an integer multiple of the natural period. Inherently, vibration induced by other possible higher flexible modes close to an integer multiple of the considered natural frequency will be damped.

One note that the considered negative input shaper with a length of one natural period of vibration is clearly not a time optimal solution considering the different possibilities offered by input shaping methodology, which are summarized in Singhose (2009). The classical Zero-Vibration Shaper length is half a period of the considered mode. The Zero-Vibration-Derivative shaper increases the insensitivity, but its time length is one period of the mode. Others shapers, including negative input shaper, can be developed to increase robustness and/or to reduce the rise time. Fig. 3 illustrates the residual vibration function $V_{J L}$ of the jerklimited command compared to the ZV- and ZVD-shaped commands. Here, $100 \%$ residual vibration corresponds to the vibration induced by a step command, and robustness or sensitivity is classically defined as the frequency range over which a command induces less than $5 \%$ vibration. These sensitivity curves reveal how much residual vibration will exist when there is an error in the estimation of the system frequency. Hence, jerk-limited trajectory tune to cancel the residual vibration of one flexible mode will be around $40 \%$ more robust than a $\mathrm{ZV}$ shaper. In counterpart, the

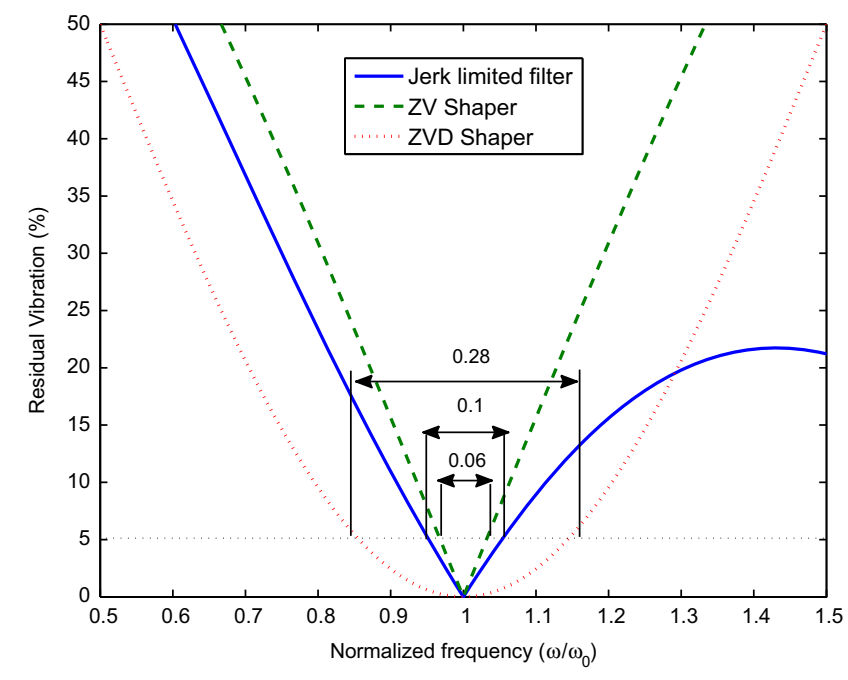

Fig. 3. Jerk-limited filter sensitivity curves.

jerk-limited filter is half a period of vibration longer. ZVD shaper will introduce the same delay, but with a significant increase of the insensitivity ( $280 \%$ more robust than the jerk-limited profile).

The real benefit of using a jerk-limited filter is induced by the low-pass filtering effect (the integrator), which naturally reduced the influence of additional neglected flexible modes. Fig. 4 illustrates the acceleration response of a two low-damped modes system with frequencies of $10 \mathrm{~Hz}$ and $25 \mathrm{~Hz}$. It can be seen that the ZV-shaped and ZVD-shaped step commands eliminate the first vibratory mode, but there is noticeable residual vibration at the second one. Such shapers can naturally damp higher vibration mode if the corresponding frequency is an odd integer of the dominant one. In other cases, multimode input shaper can be efficiently designed as depicted in Diaz et al. (2010), but at the expense of a more complex synthesis. Therefore, the low-pass filtering effect of the jerk-limited command eliminates most of the residual vibration induced by higher flexible modes, without any specific knowledge of their frequency characteristics. This effect is of particular interest in motion systems (manipulator and industrial robot), for which a lot of flexible modes, even if less sensitive, difficult to isolate and identify, contribute to the system dynamics.

However, the good results in vibration reduction obtained with the jerk-limited profile have to be relativized when the dominating flexible mode is not lightly damped. Fig. 5 presents the evolution of the residual vibration amplitude of a jerk-limited filter according to the damping of the considered flexible mode. Clearly, when the damping is above $5 \%$, the maximum residual vibration is kept under $5 \%$, but as soon as the damping is greater, the residual vibration increase significantly to reach around $20 \%$. The jerk-limited profile cannot cancel the residual vibration for an underdamped system, which is unsatisfying for the command of industrial systems and even more for the command of actively or passively damped systems.

In the next section, an evolution of the jerk profile is proposed to take account of the damping value of the considered flexible mode.

\section{New Damped-Jerk trajectory}

\subsection{Principle}

The proposed Damped-Jerk profile is based on simple considerations. According to the previous analysis (see Fig. 2), the acceleration profile of a JL motion can be deconvoluted into an adapted acceleration limited profile, a time integral and a negative 

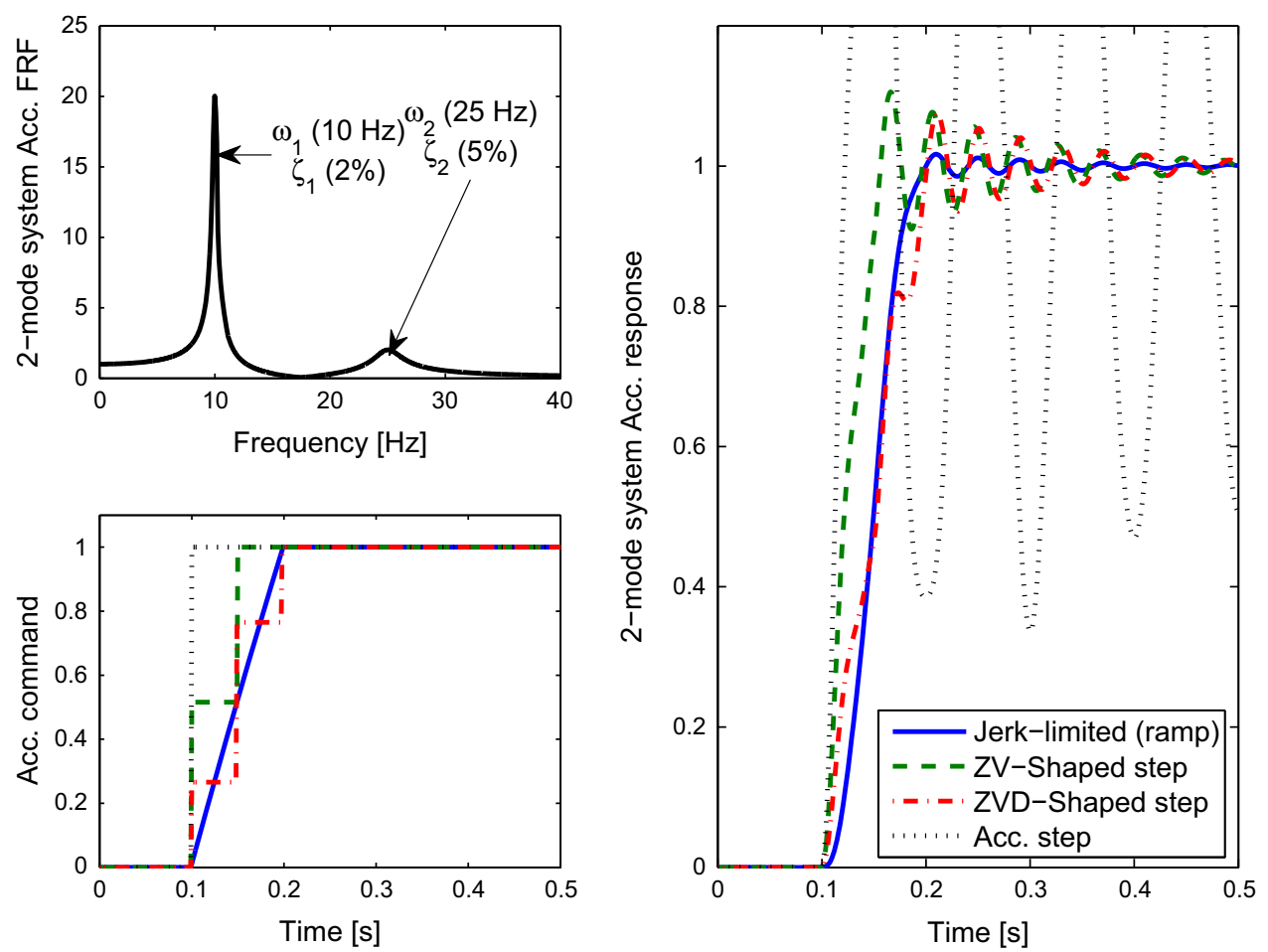

Fig. 4. Two-mode system response.
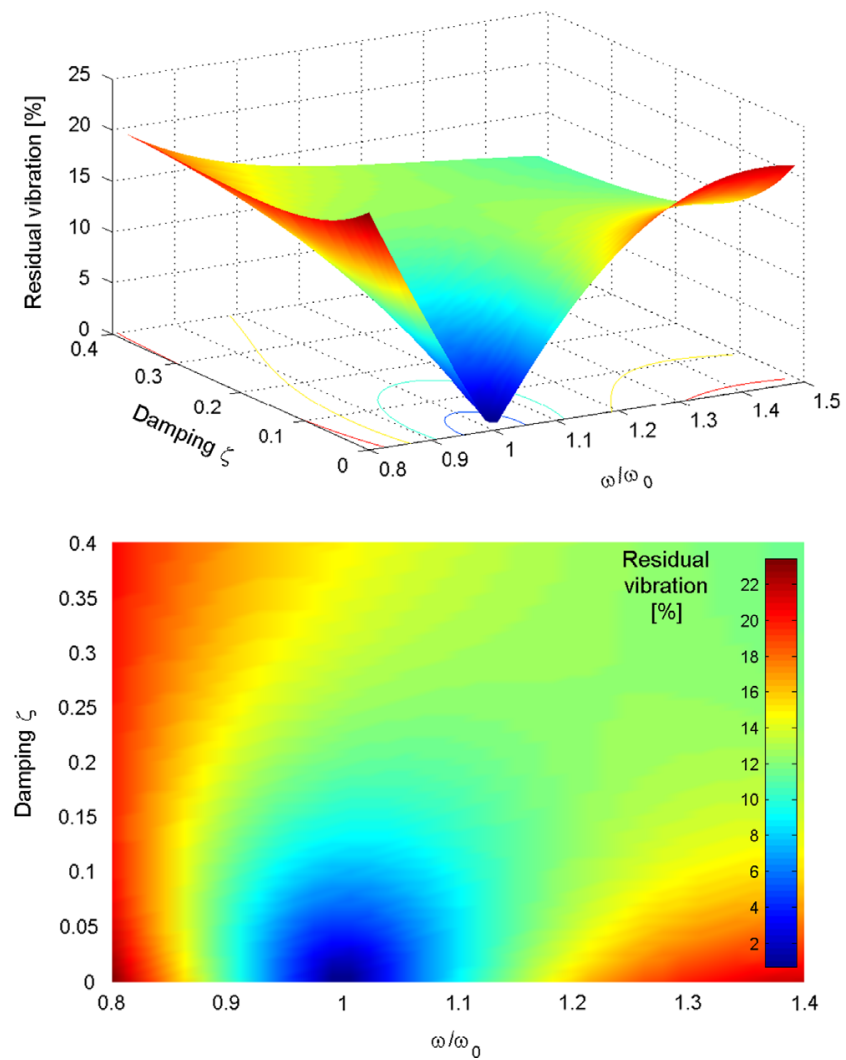

Fig. 5. Sensitivity of the jerk-limited filter according to the damping value.

two-pulse shaper. The considered negative two-pulse shaper is composed of two impulses with the same amplitude, but with the opposite sign. The equality constraint on the two impulses amplitudes is responsible for the inability to perfectly cancel damped flexible mode. Based on the input shaping methodology, to take account of the damping coefficient value, the second impulse amplitude has to be different from the first one. Indeed, considering a damped flexible mode at a frequency of $\omega_{1}$, with a damping coefficient $\zeta$, and solving the algebraic equation (3) for the flexible poles locations $s=-\zeta \omega_{1} \pm j \omega_{1} \sqrt{1-\zeta^{2}}$ leads to the set of solutions:

$$
\begin{gathered}
T_{J}=k \frac{2 \pi}{\omega_{1} \sqrt{1-\zeta^{2}}} \\
A_{1}+A_{2} e^{k 2 \pi \zeta_{1} / \sqrt{1-\zeta^{2}}}=0
\end{gathered}
$$

which implies that the amplitudes have to verify $A_{1} \neq A_{2}$. The sum of the amplitudes is different from zero, this shaper leads to a jerk pattern with piecewise constant jerk values always different from zero. Obviously, such a profile with no possibility of a constant acceleration stage cannot fulfill the kinematic constraints of the initial acceleration profile. The basic idea developed in this paper consists in taking advantage of the previous property of the negative two-pulse shaper by introducing an asymmetry into the jerk shape. Hence, the proposed Damped-Jerk profile, noted DJ profile, will be the result of the combination of two jerk steps with different amplitudes, but linearly linked by a ramp with a negative slope. Fig. 6 depicts the DJ profile and its deconvolution into equivalent shapers, which conduct here to the time integral of the sum of a two-pulse negative shaper (the two jerk steps with different amplitudes) with the integral of another two-pulse negative shaper (the jerk ramp). Adopting the notation of Fig. 6, the equivalent filter $F_{D J}$ is given by

$F_{D J}(s)=\frac{1}{S}\left(A_{1}+A_{2} e^{-T_{J} S}+\frac{1}{S}\left(B_{1}+B_{2} e^{-T_{J} s}\right)\right)$.

The second shaper impulse value $\left|B_{i}\right|$ being the slope of the jerk ramp is directly linked to the first shaper impulses values $A_{i}$ by the 


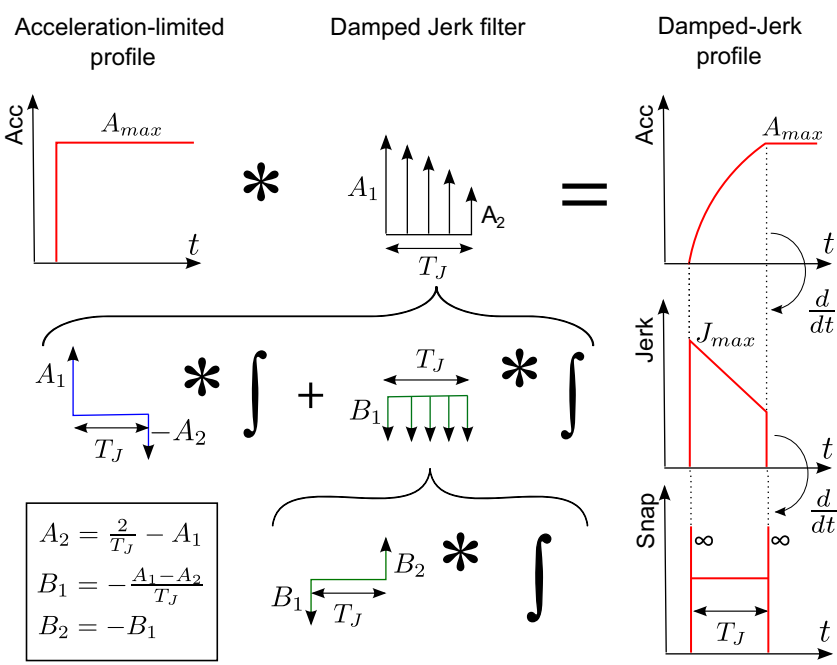

Fig. 6. Smooth input shapers associated to the proposed Damped-Jerk profile.
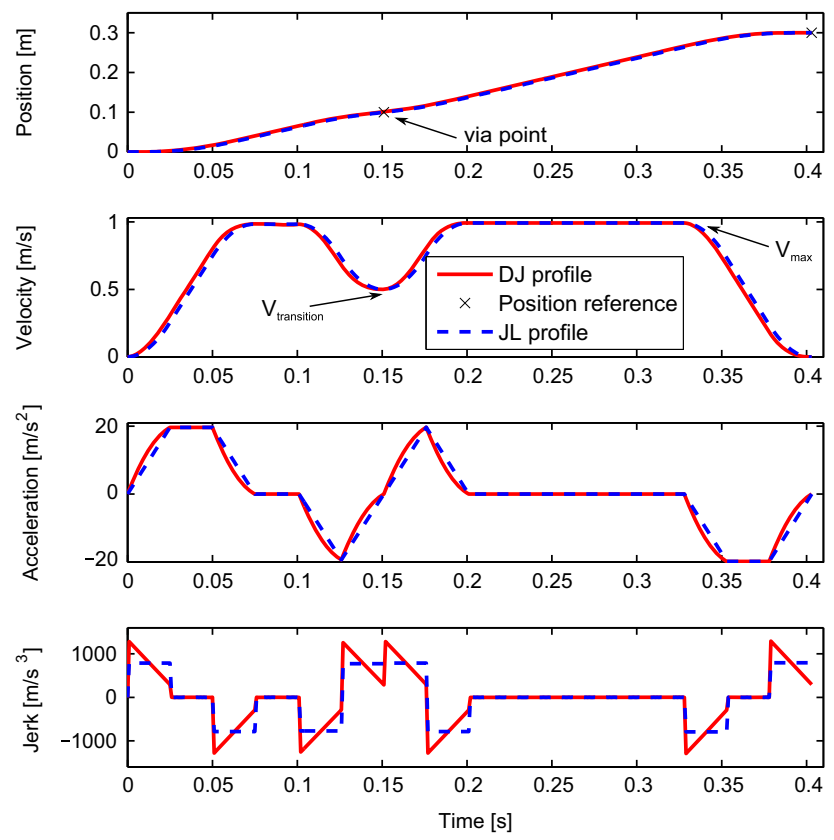

Fig. 7. Example of the DJ profile compared to the classical JL profile for via-points motion (kinematic constraints: $V_{\max }=1 \mathrm{~m} / \mathrm{s}, V_{\text {transition }}=0.5 \mathrm{~m} / \mathrm{s}, A_{\max }=20 \mathrm{~m} / \mathrm{s}^{2}$, $\left.T_{J}=20 \mathrm{~ms}\right)$.

geometric relation

$B_{1}=-B_{2}=-\frac{A_{1}-A_{2}}{T_{J}}$.

To satisfy the requirement that the final acceleration value of the DJ time-delay filter be unity when it is driven by a unit acceleration step input results in the constraint equation

$A_{1}+A_{2}=\frac{2}{T_{J}}$.

The tuning methodology of the DJ profile will be discussed in the next part. One notes that the resulting DJ profile can be easily implemented in the current controller using an average ponderated moving filter. Fig. 7 illustrates the result of the convolution of the proposed filter with an acceleration limited profile for a via points motion with constraint on the velocity transition at via point. As mentioned before, the initial acceleration limited profile takes account of the filter time $T_{J}$ as detailed in Béarée and Olabi (2013).

\subsection{Vibration reduction performances}

The amplitude of residual vibration for the Damped-Jerk profile can be calculated as follows. Considering the decomposition into equivalent shapers, as described in Fig. 6, the Damped-Jerk profile can be seen as an additive combination of a negative input shaper with amplitudes noted $A_{i}$ with the integer of another negative input shaper with amplitudes noted $B_{i}$. This equivalent filter is then integrated one more time to give the Damped-Jerk filter. The amplitude of residual vibrations results from the superposition of each filter contribution. To express residual vibration for the DJ profile, we need the expression of the response of a second-order harmonic oscillator from a sequence of steps with amplitude $B_{i}$, i.e. the time integral of $(7)$ noted $y_{\text {step }}(t)$

$y_{\text {step }}(t)=\sum_{i=1}^{n} \frac{-B_{i}}{\sqrt{1-\zeta^{2}}} e^{-\zeta \omega_{1}\left(t-T_{i}\right)} F_{i}(t)$

$F_{i}(t)=\sqrt{1-\zeta^{2}} \cos \left(\omega_{1 d}\left(t-T_{i}\right)\right)+\zeta \sin \left(\omega_{1 d}\left(t-T_{i}\right)\right)$.

Then, using trigonometric identities on the sum of (7) and (17) for $n=2$ and integrating one more time the result (division by $\omega_{1}$ ) give the following expression for the DJ profile percentage of residual vibration evaluated at $t=T_{J}$ :

$$
\begin{aligned}
& V_{D J}\left(\omega_{1}\right)=\frac{e^{-\zeta \omega_{1} T_{J}}}{\omega_{1}} \sqrt{\left(C_{D J}\right)^{2}+\left(S_{D J}\right)^{2}} \\
& C_{D J}=\sum_{i=1}^{2} e^{\zeta \omega_{1} T_{i}}\left(\left(A_{i}-\frac{\zeta B_{i}}{\omega_{1}}\right) \cos \left(\omega_{1 d} T_{i}\right)-\frac{B_{i}}{\omega_{1}} \sqrt{1-\zeta^{2}} \sin \left(\omega_{1 d} T_{i}\right)\right)
\end{aligned}
$$
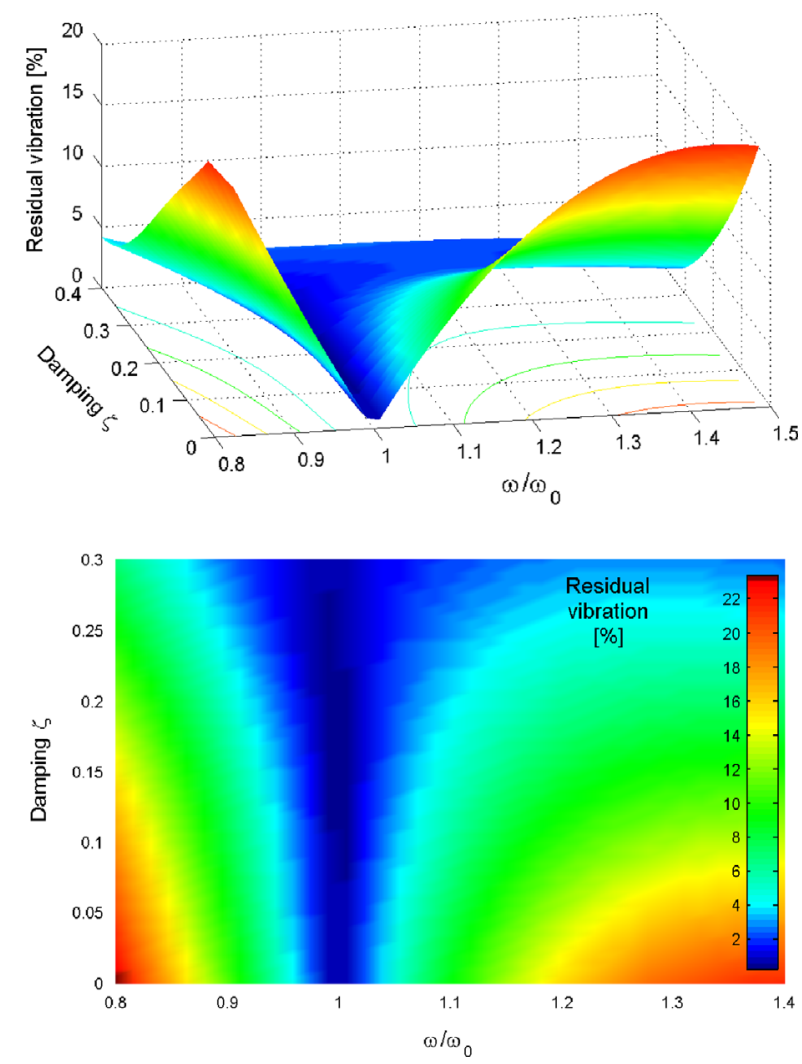

Fig. 8. Sensitivity plot of the DJ profile according to the damping value. 
$S_{D J}=\sum_{i=1}^{2} e^{\zeta \omega_{1} T_{i}}\left(\left(A_{i}-\frac{\zeta B_{i}}{\omega_{1}}\right) \sin \left(\omega_{1 d} T_{i}\right)+\frac{B_{i}}{\omega_{1}} \sqrt{1-\zeta^{2}} \cos \left(\omega_{1 d} T_{i}\right)\right)$

The second shaper associated to the ramp of jerk is symmetrical, thus from a theoretical point of view, the DJ filter cannot totally suppress damped vibration. But a good choice of parameters can significantly reduce the residual vibration. The parameters $B_{1}, B_{2}$ and $A_{2}$ being linked to $A_{1}$, thanks to the relations (15) and (16), the problem consists in finding the set of the two parameters $\left(A_{1}, T_{J}\right)$, which minimize both the amplitude of residual vibration given by (19) and the filter time $T_{J}$. The exact solution of this minimization problem cannot be expressed in closed form, but extensive numerical computations have been conducted for the approximated rules

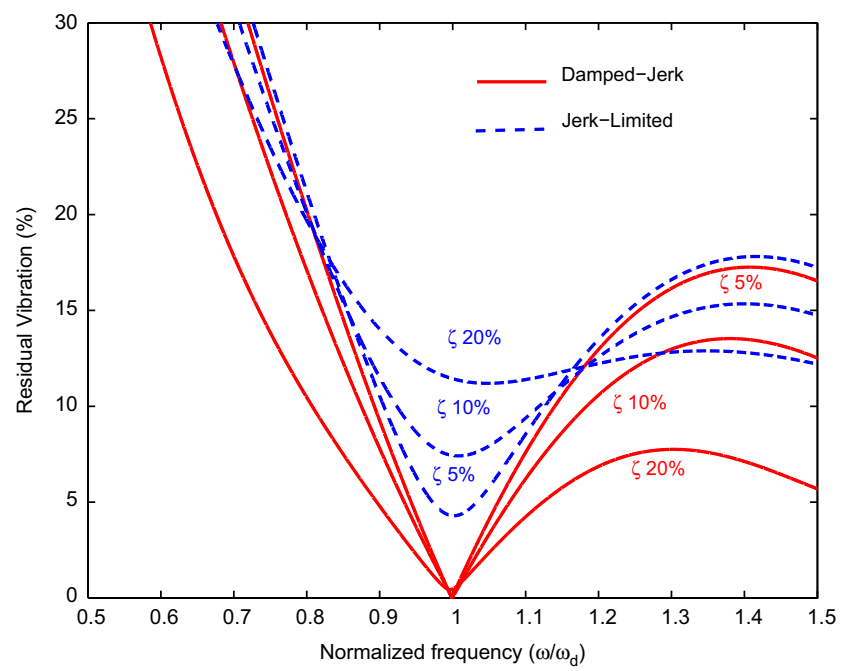

Fig. 9. Comparison of the sensitivity plots of the DJ and JL profiles according to the damping value. given by (22). We verify that for an undamped flexible mode, the relation (22) leads to the classical JL profile, with filter time equal to the natural period of the mode.

$T_{J}=\frac{2 \pi}{\omega_{1}}\left(1+0.083 \zeta+0.047 \zeta^{2}+7.1 \zeta^{3}\right)$

$A_{1}=\frac{1+\pi \zeta}{T_{J}}$

Based on the tuning rules given by (22), Fig. 8 shows the residual vibration sensitivity of the DJ profile according to the damping coefficient. Fig. 9 compared the sensitivity plots of the DJ and JL profiles for three different values of damping and Fig. 10 and 11 present the acceleration responses of a second order system for damping values of $5 \%$ and $20 \%$. One can note that the proposed DJ profile reduces significantly (below $1 \%$ ) the residual vibration around the flexible mode frequency for all the damping values. Considering inevitable numerical errors due to the time sampling, the previous theoretical result is comparable with that of an input shaper perfectly tuned to totally suppress vibration. Hence, the relation (22) allows engineer to design a DJ filter without requirement of complex numerical solving.

\section{Experiment}

\subsection{Experimental setup}

In order to clearly show the practical improvement achieved by using the proposed DJ profile, experiments were carried out on an industrial 6-axis robot depicted in Fig. 12. Industrial robots are known to be repeatable within $0.1 \mathrm{~mm}$. The static and dynamic accuracy is far beyond, typically around some millimeters. The dynamic accuracy is mainly deteriorated by joints deformation, which induced low-damped vibrations of the tip of the robot. Hence, we have chosen an industrial robot for the tests to demonstrated that, even if the vibration is very low-damped

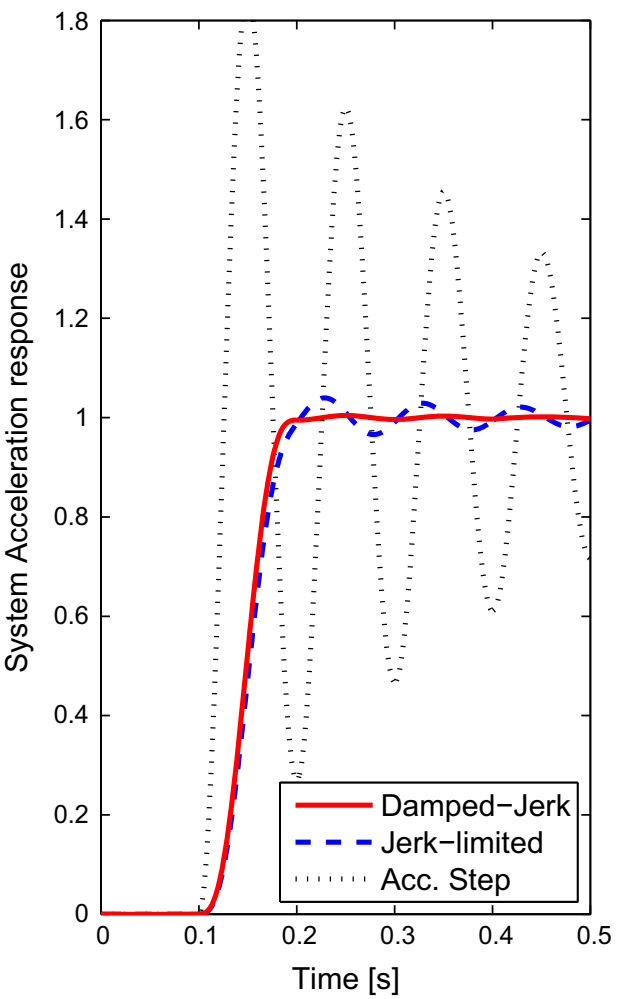

Fig. 10. DJ profile response of a second order system for a damping value of $5 \%$. 

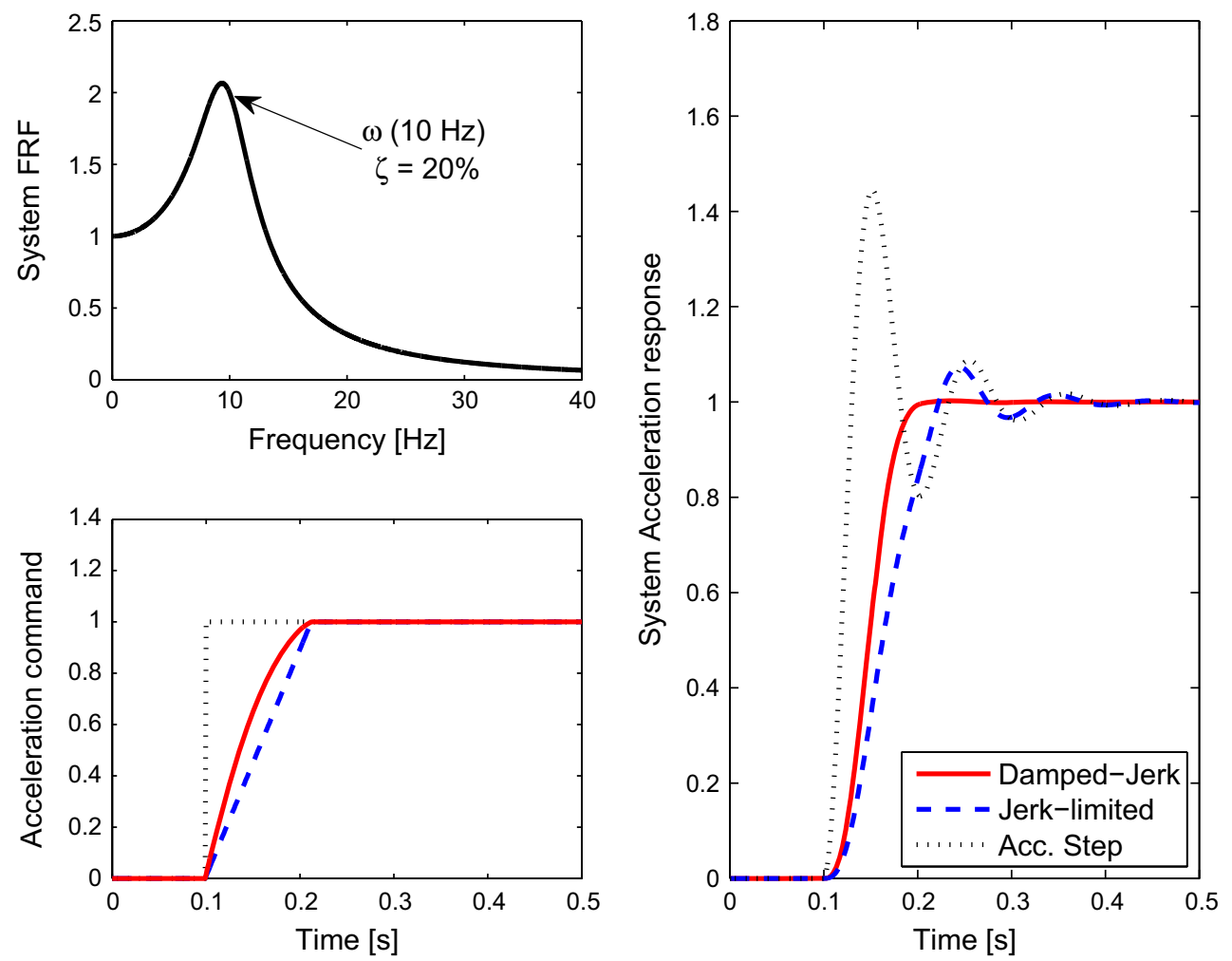

Fig. 11. DJ profile response of a second order system for a damping value of $20 \%$.
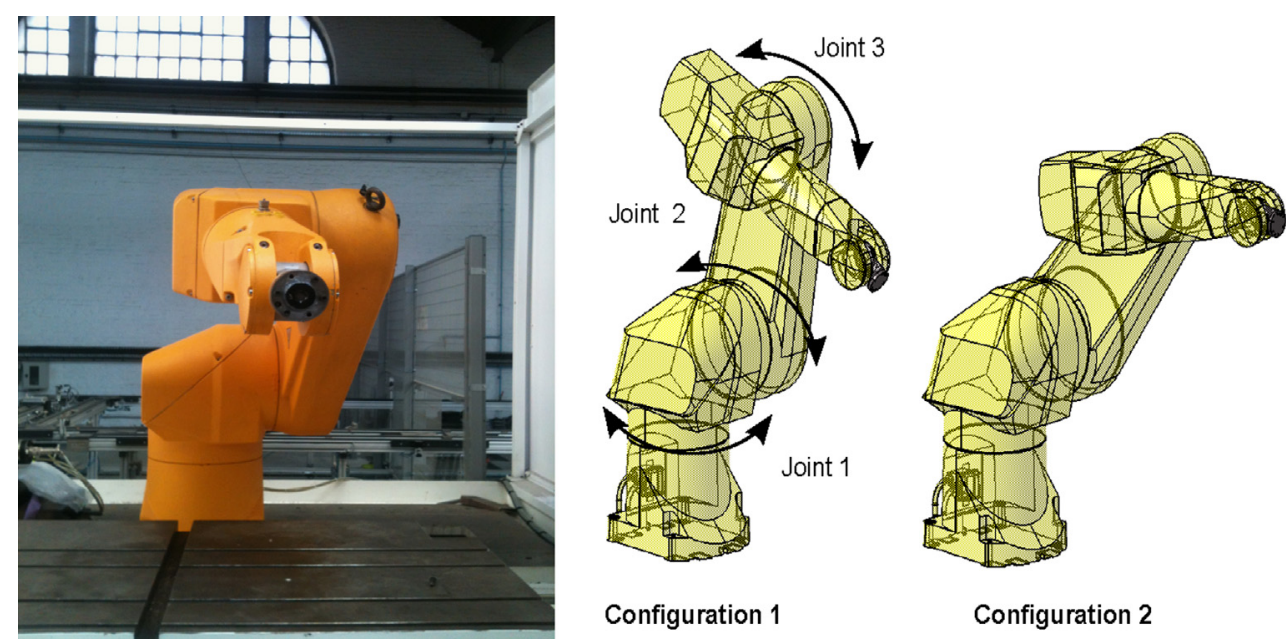

Fig. 12. Industrial robot Staubli RX170 and the two joint configurations used for the tests.

(below 7\%), the new DJ profile performances can substantially increase the system accuracy and productivity as compared to the well-tuned JL profile available in robot controllers.

For the following experiments, the motions of joint 1 of the robot are studied for two different configurations of other joints (see Fig. 12). The axis 1 traveled for $T$ second to reach the joint reference $\theta_{1 \text { ref }}$ and the tip response after $T$ is regarded as the residual vibration. The reference trajectories given by the kinematic constraints $\theta_{1 \mathrm{ref}}=0.523 \mathrm{rad}, V_{\max }=0.25 \mathrm{rad} / \mathrm{s}$ and $A_{\max }=$ $4.8 \mathrm{rad} / \mathrm{s}^{2}$ are sent to the robot controller at a sampling frequency of $250 \mathrm{~Hz}$. The tip position is measured by a laser tracker, from API Inc., with an absolute accuracy of $\pm 15 \mu \mathrm{m} / \mathrm{m}$ and a sampling frequency of $333 \mathrm{~Hz}$. Prior to the application of JL and DJ profiles, we have examined the characteristics of the vibrations of joint 1 for the two configurations of the robot. These tests give the reference values for the input filters tuning. For the first configuration of the robot, the estimated dominating frequency $\omega_{1}$ is $51.52 \mathrm{rad} / \mathrm{s}(8.2 \mathrm{~Hz})$. For the second configuration, which induces a higher equivalent inertia on joint 1 , the dominating frequency shifts to the value of $37 \mathrm{rad} / \mathrm{s}(5.9 \mathrm{~Hz})$. The estimated damping ratio was $6.5 \%$ for both configurations. One note that for the following tests, the trajectory filters are tuned on the previously estimated frequency. For the practical application on industrial robot, the proposed methodology can be advantageously combined with the real-time identification of the dominating modal frequency. Different methods described in Park et al. (2006) or Pereira, Trapero, Diaz, and Feliu (2012) can be used.

\subsection{Results}

Fig. 13 shows the experimental residual response for the two configurations of the robot and Table 1 gives the values of the 
a
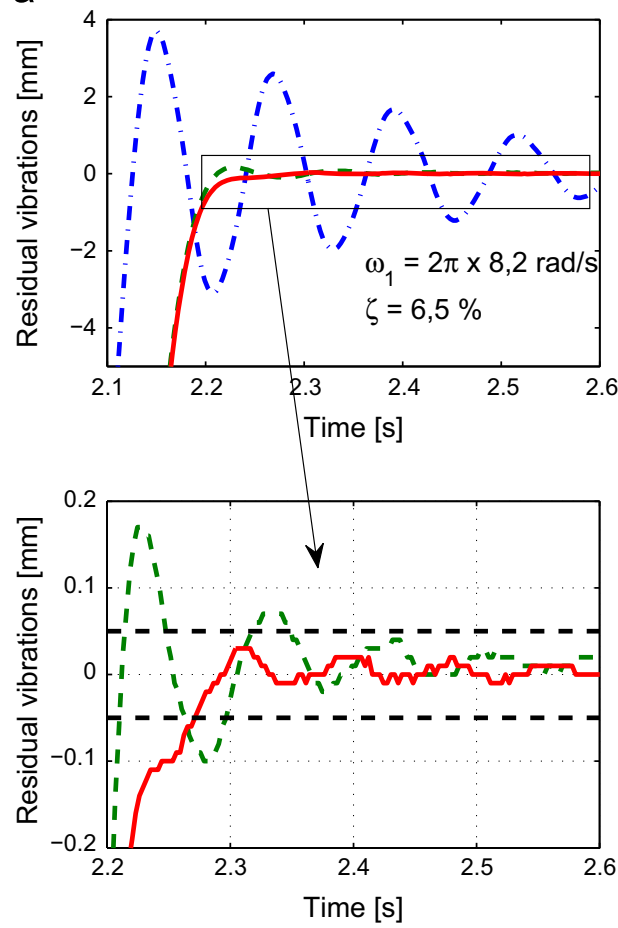

b

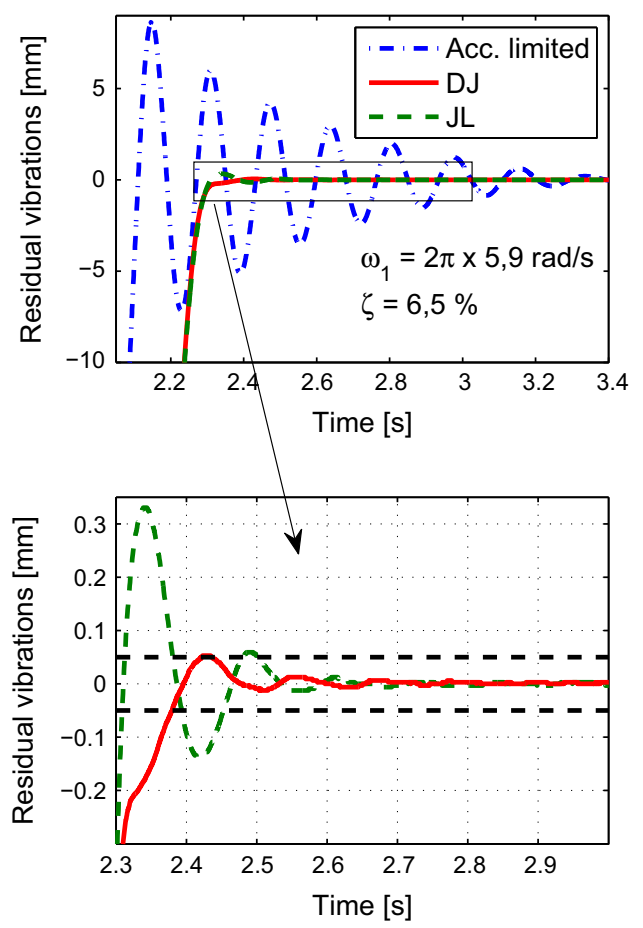

Fig. 13. Residual vibrations: (a) configuration 1 and (b) configuration 2.

Table 1

Measured maximum peak-to-peak vibration.

\begin{tabular}{lll}
\hline Trajectory profile & Configuration 1(mm) & Configuration 2 (mm) \\
\hline Acc. limited & 6.7 & 15.8 \\
Jerk-limited & 0.29 & 0.47 \\
Damped-Jerk & 0.04 & 0.06 \\
\hline
\end{tabular}

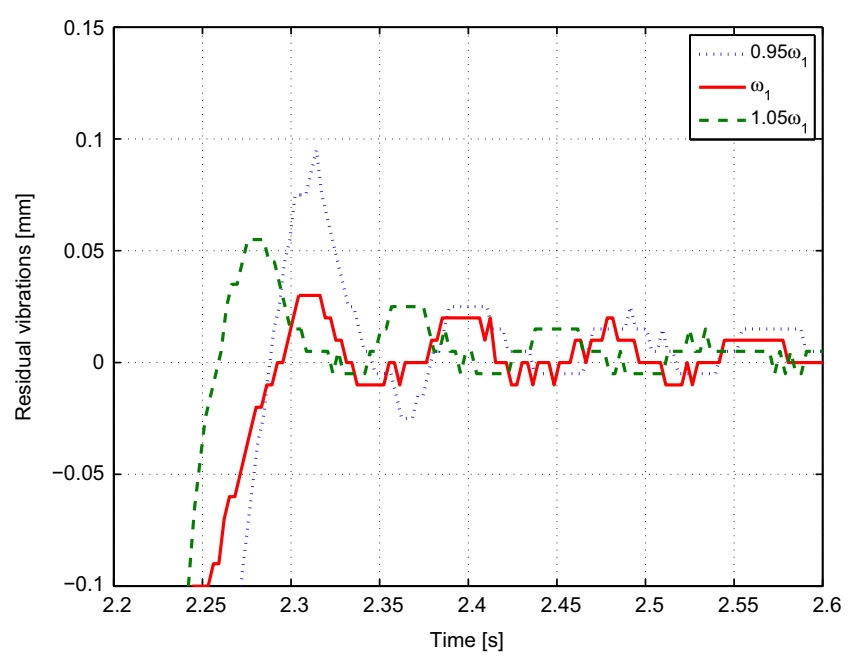

Fig. 14. Residual vibration of the DJ profile according to the estimated frequency error.

maximum peak-to-peak residual vibration for each trajectory profiles. The measured vibrations clearly show the huge improvement induced by JL and DJ profiles. For both configurations, the well-tuned JL profile has reduced the residual vibration to $4 \%$ and the DJ profile to less than $0.5 \%$ of the initial level. In order to demonstrate the effectiveness of the proposed tuning methodology for the DJ profile (given by (22)), Fig. 14 shows the tip residual response according to the error on the estimated frequency. The local minimum of vibration induced by the DJ profile is unmistakably at the estimated value of $\omega_{1}$.

Now, considering the settling time for a tolerance band of $0.1 \mathrm{~mm}$ (repeatability of the robot), the DJ profile conducted to a faster response than the JL profile with a gain of more than $0.1 \mathrm{~s}$ ( $5 \%$ of the theoretical motion time). These results demonstrate that even for low-damped vibrations, the proposed DJ profile can be conducted to a noticeable improvement in industrial system performances.

\section{Conclusions}

A simple technique to design the jerk-shaped profile adapted to damped vibrations reduction is proposed in this paper. The jerk-limited profile ability to cancel undamped vibrations is first addressed. To explain this result, the classical JL profile used by industrial motion systems is decomposed into equivalent filtered Input shapers. This methodology motivates the design of a new jerk-shaped profile, named Damped-Jerk profile, which extends the previous result to the more general case of underdamped vibrations. Practical tuning rules for the equivalent input filter parameters are then given. Experimental tests conducted on an industrial robot axis demonstrated the effectiveness of the proposed methodology and clearly show the benefits offered by using the DJ profile even in the case of low-damped vibrations.

One can conclude that such a practical methodology naturally combines with respect to a kinematic constraint on maximum velocity and acceleration, with the potential of vibration cancellation for the dominating flexible mode and the smoothness of trajectory for reduction of the contribution of neglected or unstationary flexible modes. Hence, it can be seen as an interesting alternative to classical input shaping technics for industrial systems dedicated to fast and accurate positioning or trajectory following. 


\section{References}

Béarée, R. \& Olabi, A. (2013). Dissociated jerk-limited trajectory applied to timevarying vibration reduction. Robotics and Computer-Integrated Manufacturing 29(2), 444-453.

Biagiotti, L., \& Melchiorri, C. (2012). FIR filters for online trajectory planning with time- and frequency-domain specifications. Control Engineering Practice, 20(12) 1385-1399.

Diaz, I. M., Pereira, E., Feliu, V., \& Cela, J. J. L. (2010). Concurrent design of multimode input shapers and link dynamics for flexible manipulators. IEEE/ASME Transactions on Mechatronics, 15(4).

Kim, J.J., Agrawal, B.N. (2006). Experiments on jerk-limited slew maneuvers of a flexible spacecraft. In Proceedings of the AIAA Guidance, Navigation, and Control Conference and Exhibit, August.

La-orpacharapan, CH., \& Pao, L. Y. (2005). Fast and robust control of systems with multiple flexible mode. IEEE/ASME Transactions on Mechatronics, 10(5).

Olabi, A., Béarée, R., Gibaru, O., \& Damak, M. (2010). Feedrate planning for machining with industrial six-axis robot. Control Engineering Practice, 18(5), 471-482.

Park, J., Chang, P. H., Park, H. S., \& Lee, E. (2006). Design of learning input shaping technique for residual vibration suppression in an industrial robot. IEEE/ASME Transactions on Mechatronics, 11(1).
Peng, K. C. C., Singhose, W., \& Frakes, D. H. (2012). Hand-motion crane control using radio-frequency real-time location system. IEEE/ASME Transactions on Mechatronics, 17(3).

Pereira, E., Trapero, J. R., Diaz, I. M., \& Feliu, V. (2012). Adaptive input shaping for single-link flexible manipulators using an algebraic identification. Control Engineering Practice, 20(2), 138-147.

Piazzi, A., \& Visioli, A. (2000). Minimum-time system-inversion-based motion planning for residual vibration reduction. IEEE/ASME Transactions on Mechatronics, 5(1).

Singer, N. C., \& Seering, W. P. (1990). Preshaping command input to reduce system vibration. Journal of Dynamics, System, Measure and Control, 112, 76-82.

Singh, T. (2004). Jerk limited input shapers. ASME Journal of Dynamic Systems Measurement and Control, 126(1), 215-219.

Singhose, W. (2009). Command shaping for flexible systems: A review of the first 50 years. International Journal of Precision Engineering and Manufacturing, 10(4), 153-168.

Singhose, W., \& Pao, L. (2009). A comparison of input shaping and time-optimal flexible-body control. Control Engineering Practice, 5(4), 459-467.

Smith, O. J. M. (1957). Posicast control of damped oscillatory systems. Proceedings of the IRE, 45(4), 1249-1255. 